\title{
Transcatheter aortic valve replacement: a further step towards a patient-tailored therapy
}

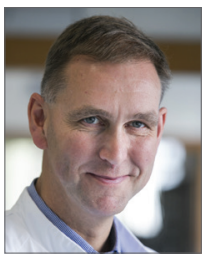

Johan Bosmans ${ }^{1}$, MD, PhD; Bernard Paelinck ${ }^{2}$, MD, PhD

1. Department of Cardiology, Interventional Cardiology, University Hospital Antwerp, Antwerp, Belgium;

2. Department of Cardiology, Non-invasive Imaging, University Hospital Antwerp, Antwerp, Belgium

Transcatheter aortic valve replacement (TAVR) is a rapidly evolving technique providing robust treatment of severe aortic valve stenosis, at least in (very) high-risk patient cohorts. However, different important limitations, having a potential impact on patient outcome, still need to be resolved. Indeed, with several firstgeneration balloon-expandable or self-expanding TAVR prostheses, residual paravalvular aortic regurgitation (AR) after TAVR has been identified as a significant independent major predictor of acute and long-term mortality. Moreover, conduction disorders requiring pacemaker implantation, and also concerns about early (leaflet thrombosis/decreased leaflet motion) and late valve durability, need to be resolved before applying TAVR to lower-risk patient cohorts. These limitations appear to be partially patient, but certainly also TAVR system specific.

In this issue of EuroIntervention, safety and efficacy at 30 days of two new-generation TAVR prostheses (the resheathable and repositionable Portico ${ }^{\mathrm{TM}}$ self-expanding valve' ${ }^{1}$; St. Jude Medical, Article, see page 768

St. Paul, MN, USA ${ }^{1}$ and the fully repositionable Lotus ${ }^{\mathrm{TM}}$ valve $^{2}$; Article, see page 760

Boston Scientific, Marlborough, MA, USA) are presented in small prospective series. Both valves add to the armamentarium of current TAVR systems in the pursuit of a patient-tailored therapy. Although in these studies high-quality data of TAVR in high-risk patients are presented, some aspects need additional attention.

Resheathing or repositioning in order to optimise the final position of the valves was done in roughly one in every four patients with each of the frames. From the available data, it seems that this was able to be carried out safely and effectively, although with Portico mean stent protrusion into the left ventricular outflow tract for patients who had resheathing was still $6 \mathrm{~mm}$, reflecting a relatively deep final valve implantation. Moreover, also using the Portico frame, there were four patients who received two valves despite the possibility of resheathing (3.9\%). These results are similar to those in the CoreValve ADVANCE study, in which a self-expanding non-resheathable system was investigated, and where $4 \%$ of patients ended up with two valves. Additional points of concern are the obligatory increased use of potentially nephrotoxic contrast media together with prolongation of the procedural time. Although this may be the price to pay for one or more additional attempts to reposition the valve, it remains a clinically potentially relevant issue in a significant number of patients with renal insufficiency. Finally, the risk of increasing neurological events by additional manipulations in severely calcified native aortic valves in order to improve final valve position is not yet completely excluded based on the data of the currently available relatively small studies.

Reducing the occurrence of major conduction disturbances and preventing paravalvular leakage seem to be somewhat "contradictory" goals also for these newer-generation TAVR devices. While in the Portico series a range of small valves (19-23 mm) was implanted in high-risk, mainly female (97\%) patients, in the Lotus series a greater range $(20-27 \mathrm{~mm})$ was implanted $(50.9 \%$ female in total; $95 \%$ female in small valves), requiring pacemaker

*Corresponding author: Department of Cardiology, University Hospital Antwerp, Antwerp, Belgium.

E-mail:johan.bosmans@uantwerpen.be 
implantation in $9.8 \%$ and $24.1 \%$, respectively. On the other hand, residual moderate $(5.1 \%)$ and severe $(1.2 \%)$ paravalvular insufficiency in the Portico series as compared with the Lotus series $(0 \%)$ is an interesting observation. The specific design of the TAVR valve and its size may increase the risk of mechanical compression on the atrioventricular conduction system but conversely decrease the risk of paravalvular aortic regurgitation. The extremely low incidence of moderate or severe paravalvular insufficiency using Lotus is in line with previous data from the REPRISE study ${ }^{3}$. The prospective, single-arm, multicentre REPRISE II and REPRISE II Extension studies enrolled 250 symptomatic, high surgical risk patients with calcific aortic stenosis and an annulus of 19-27 mm. Paravalvular regurgitation was assessed by echocardiography at discharge and 30 days according to VARC-2 criteria, and aortic valve dimensions and calcification by $\mathrm{CT}$ scan in end systole; both were analysed and reported by an independent core lab. At 30 days, $80.2 \%(142 / 177)$ of patients had no paravalvular regurgitation, $5.6 \%(10 / 177)$ had trace/trivial regurgitation, $13.6 \%$ (24/177) had mild regurgitation, $0.6 \%$ (1/177) had moderate regurgitation, and no patients had severe paravalvular regurgitation. Therefore, pooling the evidence we actually have, paravalvular aortic regurgitation rates are clearly low overall with the Lotus valve and consist almost exclusively of mild paravalvular leakage. A relatively high pacemaker need after Lotus implantation, however, again seems to be the price to pay. Long-term prognosis in the Lotus series, with no moderate/severe aortic regurgitation reported, is of the utmost interest.

Recently, in the prospective, multicentre, randomised trial (transcatheter versus surgical aortic valve replacement) in intermediate-risk patients with severe aortic stenosis (PARTNER II), Leon et $\mathrm{al}^{4}$ confirmed again that moderate to severe (paravalvular) aortic regurgitation remains a major determinant of morbidity and mortality following TAVR and occurs in up to $24 \%$ of patients. Therefore, in order to quantify adequately the severity of aortic regurgitation after TAVR, and also to make a fair comparison between different TAVR frames concerning the severity of residual regurgitation, we urgently need validated uniform quantification criteria. Indeed, an important pending issue is how to quantify paravalvular regurgitation most adequately. Aortic regurgitation after TAVR can be assessed using angiography, echocardiography and/or haemodynamics (Figure 1). Although recently published guidelines offer several techniques to evaluate postTAVR aortic regurgitation, practical limitations specifically related to correct evaluation of (paravalvular) leakage complicate correct quantification and regularly lead to discordant results. Moreover, prognostically validated quantification criteria of aortic regurgitation after TAVR, for each of these techniques, have not yet been clearly defined. Finally, to the best of our knowledge, studies comparing angiography, echocardiography and invasive haemodynamics (aortic regurgitation index post) are relatively scarce. Periprocedural echocardiography for the grading of paravalvular regurgitation is extremely challenging despite using an integrative approach, due to acoustic reverberations of the prosthetic material and/or calcifications, eccentricity and/or multiplicity of regurgitation jets. In PARTNER II, the expanded grading scheme proposed by Pibarot et $\mathrm{al}^{5}$ overcomes several of these shortcomings but still needs validation. Collas et $\mathrm{al}^{6}$ used a simplified reproducible colour Doppler approach to assess aortic regurgitation area. This simplified method predicted one-year mortality. Furthermore, further study is needed to assess volumetric quantification of blood flow with three-dimensional echocardiography.

As mentioned previously, concerns about early and late valve durability need to be resolved before TAVR can be applied safely to lower-risk patient cohorts. Reduced leaflet motion as a marker of subclinical leaflet thrombosis ${ }^{7}$ and the effect of anticoagulation on the risk of stroke are ongoing issues. The potential impact of

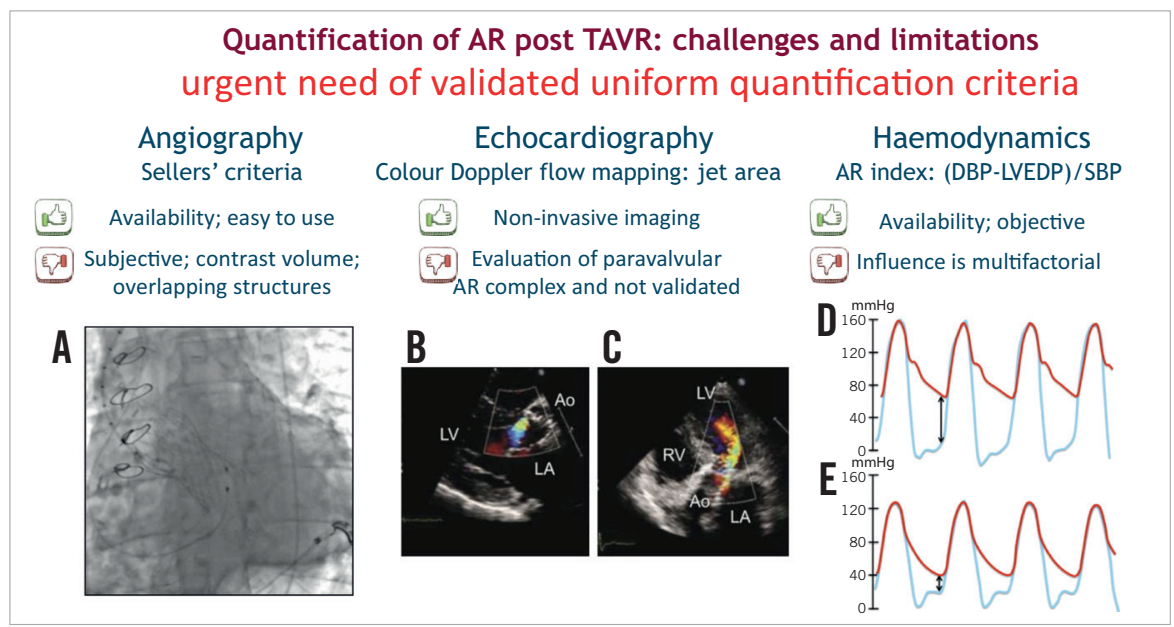

Figure 1. Evaluation of AR post TAVR. Main advantages and disadvantages of angiography (A); echocardiography, including colour Doppler in parasternal long-axis (B) and apical 5-chamber view (C); and invasive haemodynamics, high AR-index (D) and low AR-index or poorly tolerated AR (E). 
stent design and related flow dynamics is an unsolved question. Clear data on leaflet motion in these Portico and Lotus series are lacking but were, at least previously, a point of concern certainly for the Portico frame. For the Lotus frame, we have to take into account the somewhat higher mean gradients $(20-25 \mathrm{mmHg})$ after TAVR, compared to what we usually see with other TAVR frames.

The question of which valve design/size for which patient needs further multicentre, prospective investigation. The reported Portico and Lotus series provide one step forward to a patienttailored therapy. Imaging, including quantification of paravalvular regurgitation, and assessment of subclinical valve thrombosis are major goals in TAVR.

\section{Conflict of interest statement}

J. Bosmans is a consultant for Medtronic CoreValve. B. Paelinck has no conflicts of interest to declare.

\section{References}

1. Manoharan G, Linke A, Moellmann H, Redwood S, Frerker C, Kovac J, Walther T. Multicentre clinical study evaluating a novel resheathable annular functioning self-expanding transcatheter aortic valve system: safety and performance results at 30 days with the Portico $^{\mathrm{TM}}$ system. EuroIntervention. 2016;12:768-74.

2. Wöhrle J, Gonska B, Rodewald C, Seeger J, Scharnbeck D, Rottbauer W. Transfemoral aortic valve implantation with the repositionable Lotus valve for treatment of patients with symptomatic severe aortic valve stenosis: results from a single-centre experience. EuroIntervention. 2016;12:760-7.

3. Meredith IT, Walters DL, Dumonteil N, Worthley SG, Tchétché D, Manoharan G, Blackman DJ, Rioufol G, Hildick-
Smith D, Whitbourn RJ, Lefèvre T, Lange R, Müller R, Redwood S, Feldman TE, Allocco DJ, Dawkins KD. 1-Year Outcomes With the Fully Repositionable and Retrievable Lotus Transcatheter Aortic Replacement Valve in 120 High-Risk Surgical Patients With Severe Aortic Stenosis: Results of the REPRISE II Study. JACC Cardiovasc Interv. 2016;9:376-84.

4. Leon MB, Smith CR, Mack MJ, Makkar RR, Svensson LG, Kodali SK, Thourani VH, Tuzcu EM, Miller DC, Herrmann HC, Doshi D, Cohen DJ, Pichard AD, Kapadia S, Dewey T, Babaliaros V, Szeto WY, Williams MR, Kereiakes D, Zajarias A, Greason KL, Whisenant BK, Hodson RW, Moses JW, Trento A, Brown DL, Fearon WF, Pibarot P, Hahn RT, Jaber WA, Anderson WN, Alu MC, Webb JG; PARTNER 2 Investigators. Transcatheter or Surgical Aortic-Valve Replacement in Intermediate-Risk Patients. $N$ Engl J Med. 2016;374:1609-20.

5. Pibarot P, Hahn RT, Weissman NJ, Monaghan MJ. Assessment of paravalvular regurgitation following TAVR: a proposal of unifying grading scheme. JACC Cardiovasc Imaging. 2015;8:340-60.

6. Collas VM, Paelinck BP, Rodrigus IE, Vrints CJ, Bosmans JM. Aortic regurgitation after transcatheter aortic valve implantation (TAVI) - Angiographic, echocardiographic and hemodynamic assessment in relation to one year outcome. Int $J$ Cardiol. 2015;194:13-20.

7. Makkar RR, Fontana G, Jilaihawi H, Chakravarty $T$, Kofoed KF, de Backer O, Asch FM, Ruiz CE, Olsen NT, Trento A, Friedman J, Berman D, Cheng W, Kashif M, Jelnin V, Kliger CA, Guo H, Pichard AD, Weissman NJ, Kapadia S, Manasse E, Bhatt DL, Leon MB, Søndergaard L. Possible Subclinical Leaflet Thrombosis in Bioprosthetic Aortic Valves. $N$ Engl J Med. 2015;373:2015-24. 